\title{
Understanding user practices in mobility service systems: Results from studying large scale corporate MaaS in practice
}

\author{
Mia Hesselgren*, Martin Sjöman, Anna Pernestål \\ KTH Royal Institute of Technology, Stockholm, Sweden
}

\section{A B S T R A C T}

Mobility as a Service (MaaS), where different shared modes of transportation are bundled into one easily accessible service, plays an important role in the shift towards more sustainable transport systems. In this article, we present empirical research with the aim to understand how the barriers to increased shared travel with MaaS can be lowered. The concept of corporate MaaS (CMaaS) is introduced, and empirical results are presented from a study of CMaaS at a workplace of 14,000 employees in Sweden. The findings are based on 77 interviews with CMaaS users, performed in four iterative rounds using service design methods. Social practice theories are used as analytical lens to attempt to understand travel practices in the context of CMaaS. As CMaaS (and MaaS) are socio-technical systems, several perspectives need to be integrated in order to reach this understanding; all system components, including materials (e.g. the user application, the transport modes), competences (knowledge of how to use the materials), and meanings (understandings of travel habits, lifestyle choices, and employer relations) need to be analysed. Through this analytical lens, three barriers to adoption of CMaaS and sustainable transport were identified: inadequate integration of the internal transport system with external transport systems; corporate policy, culture and norms that conflict with using the services; and system limitations due to laws and regulations. All these barriers are also relevant for understanding MaaS services in general.

\section{Introduction}

Shared mobility, e.g. car or bike sharing, has the potential to reduce vehicle use (Fishman et al., 2014; Martin et al., 2010), congestion (Wong et al., 2017), and $\mathrm{CO}_{2}$ emissions (Fishman et al., 2014; Martin et al., 2010). Mobility as a Service (MaaS), where different shared modes of transportation are bundled into an easily accessible service, has the potential to increase use of shared modes (Ho et al., 2018) and is therefore a concept that could play an important role in the shift towards more sustainable transport systems. There are several theoretical concepts and pilots for Maas, but they have, with few exceptions, not been turned into real, fully implemented services (Ho et al., 2018). As Ho et al. (2018, p. 304) note, "the vast majority of this work [MaaS related research] constitutes think pieces and literature reviews rather than empirical research". In contrast, in this article, we present empirical research on Maas by investigating the outcomes of a Corporate MaaS system implemented by a Swedish company at an industrial estate with 14,000 employees.

In this article, we define the concept of Corporate MaaS (CMaaS), i.e. MaaS in a corporate setting. The investigated CMaaS system offers internal transport at the work site and, to a limited extent, commuting services. About twenty-five percent of the employees were active users of the CMaaS service during its first four months. The results presented are based on 77 interviews with employees, performed in four iterative rounds using a service design approach. We argue that CMaaS has much in common with MaaS in general, and it is possible to use the learnings from MaaS in the corporate context to understand MaaS in the general context. However, there are also some key differences regarding barriers and opportunities around CMaaS.

There are several potential barriers on the road to increased shared travel with MaaS, including e.g. demand modelling and understanding, integration of information from different suppliers, governance, and business models (Jittrapirom et al., 2017; Ho et al., 2018; Pernestål Brenden and Holmberg, 2017). The workplace setting provides a relationship between the users of the system that can lower the barriers to shared mobility (Sopjani et al., 2017). As employees travel to the same destination in the morning and from the same origin in the afternoon, the potential to match rides is high (Lu et al., 2018). This provides a good starting point for shared mobility, but there are indications that it is not enough (Pernestål Brenden et al., 2018). In this article, we investigate the barriers to Maas usage by studying MaaS in a corporate setting.

Understanding (and changing) travel behaviour is a complex task that often requires the involvement of several stakeholders (Banister, 2008). One of the main stakeholders is the user, but to understand them and their usage of socio-technical systems, it is also necessary to understand how the individual is related to socio-material structures (Spaargaren, 2003). Using social practice theories as analytical lens, it

\footnotetext{
* Corresponding author.

E-mail addresses: miahes@kth.se (M. Hesselgren), martsjo@kth.se (M. Sjöman), pernestal@kth.se (A. Pernestål).
} 
is possible to achieve a deeper understanding of both users and systems.

The present study contributes to the MaaS research field with knowledge about the socio-technical system of which both CMaaS and general Maas are a part. The main research question we explore in this article is: What can we learn about the design of mobility service systems through understanding user practices?

\section{Corporate MaaS}

There are several definitions and classifications of MaaS (Giesecke et al., 2016; Ho et al., 2018; Jittrapirom et al., 2017; Kamargianni et al., 2016; Matyas and Kamargianni, 2018). All agree that MaaS systems include the following components: they bring together several transport modes that are integrated using ICT and a digital platform; they have a user centric approach in the sense that they provide a "one-stop shop" of mobility; and they require some kind of registration or subscription. Definitions differ in their views of whether ticketing and payment should be integrated in the MaaS system, but when they are, it is possible to reach a higher level of integration, as discussed by Kamargianni et al. (2016).

The literature on MaaS encompasses several perspectives, e.g. user demand (Matyas and Kamargianni, 2017; Sochor et al., 2016), societal perspectives (Wong et al., 2017) and intelligent transport systems (Brendel and Mandrella, 2016; Giesecke et al., 2016). MaaS has several potential benefits for society, including e.g. reduction of congestion and land use opportunities (Wong et al., 2017), and has been put forward by Wilson et al. (2018) as one of the disruptive socio-technical systems that can contribute to solving climate challenges and reach the 1.5 degree goal for global warming set out by the Paris Agreement.

We introduce here the concept of Corporate MaaS (CMaaS), by which we mean:

- A MaaS service owned or commissioned, or in other ways controlled, by a corporation.

- A MaaS service focused on transport within, to and from a work site or campus, or between work sites or campuses. The users of the service are the people working at or visiting the site.

CMaaS is a MaaS service in the sense that it satisfies the following four criteria: it is an integration of several transport modes; it is based on a digital platform; it provides a "one-stop shop" from a user-centred perspective; and it requires registration (which may be done automatically for all employees). Furthermore, there may be different service levels (packages) including paid and free packages.

Besides offering a solution to internal transport at the work site or campus, a CMaaS solution can also be seen as a "sandbox" version of Maas in general where some of the barriers to MaaS adoption are lowered. With a limited area of operation and the organisation being able to control many of the contextual variables surrounding the service, such as ticketing and pricing variables, the corporate setting offers good prerequisites to develop a well-functioning MaaS implementation as well as learn about user practices. As transport is concentrated to the work site, and in particular some common spaces, there is a relatively high transport demand in the area and consequently many overlapping trips.

The employer's objectives are similar to those of a city: to offer sustainable transport solutions that are accessible, attractive and effective for users, and to reduce car use in order to release space and reduce congestion. However, it might be easier for an employer to steer towards these goals than it is for a city, as the company could strongly incentivise or even enforce the use of the Maas service. This would be more difficult for most cities, and should be taken into consideration when generalising results from CMaaS to general MaaS services. However, both companies and cities can work with "carrots and sticks", favouring more sustainable options if the aim is to foster sustainability transitions. Employees of an organisation have similar travel needs for commuting to a greater extent than city citizens, and employees can also more easily align with a company vision than citizens might be able to do with municipal development plans for a city.

\section{Methodological framework}

In this project, we use a design research approach, and in particular co-design and service design methods. In co-design, users are viewed as partners (Sanders, 2008). Designing product-service systems together with users as co-creators is particularly useful in generative design research, i.e. in the early stages of developing ideas of possible futures (Sanders and Stappers, 2014). With this approach, similar to action research and participatory design research, there is an agreement that all people are creative (Sanders and Stappers, 2014). Also, with participatory action research approaches, both researchers and participants are actively engaging in problem solving (Cassell and Johnson, 2006). This approach, with its inclusive methods, encourages openness and fosters collaborative research cultures. Interventions created, followed and studied in action research often have the aim to both understand situations and improve them as well as effect change. With active research approaches, there are many options for studying change, and changed user practices, such as experiments, living labs and different types of interventions (Strengers and Maller, 2014).

With service design methods, it is possible to work need-driven, hence developing user insights (Stickdorn and Schneider, 2014). Service design methods are user-centred, co-creative and iterative, and with these methods, services are designed to enhance users' experiences by integrating technologies with corporate objectives (Stickdorn and Schneider, 2014). Typically, in service design projects, three rounds of service development iterations are carried out, where the first starts very broad and explorative, the second uses trigger materials in conversations with users to reach a deeper understanding, and the third round tests a service prototype to capture user experiences. Trigger materials are used to encourage reflections and also elicit tacit knowledge. In this project, the redesigned CMaaS system, is considered such a prototype. Service design thinking is an interdisciplinary and integrative approach that has the potential to lead to the development of more sustainable solutions (Manzini and Vezzoli, 2003).

Changing behaviours to become more sustainable is far from easy, as individuals' behaviours are embedded in social contexts (Jackson, 2005). Sustainable lifestyles are therefore not only about the individual's choices in everyday life, but also the connecting systems of provisions and structures surrounding the individual (UNEP, 2016). Social practice theories can be useful to better understand lifestyles and how they are formed, e.g. through lifestyle choices. A lifestyle can be defined as a set of social practices embraced by the individual and includes the storytelling that rationalises these practices (Giddens, 1991). Using practices as a unit of analysis reveals possibilities for policy making when seeking to create changes in sustainable directions (Spaargaren, 2003). As Spaargaren (2003) explains, social practices and lifestyles are twin concepts where practices are constructed in the middle between lifestyles and systems of provision. A social practice approach puts the individual's responsibilities, and possibilities, in direct relation to socio-material structures. The systems of provision can either enable or obstruct the adoption of more sustainable lifestyles on the individual level. For organisations and system suppliers to develop these systems, an understanding of the practices related to them is required to ensure users can integrate the system with their lifestyle. A social practice approach requires a change of focus, from viewing people as individual decision makers to instead addressing them as skilful social negotiators (Shove, 2003). To focus on practices is thus to understand what people perceive as their normal ways of doing, and how ordinary routines are developed, maintained, and changed over time (Shove et al., 2007). Practices are reproduced, carried out and performed through engagements with socio-material systems, i.e. practices are scripted and structured by technologies (Shove et al., 
2007).

To understand how less resource-demanding practices can evolve, we need to consider the dynamics of practices (Spurling and McMeekin, 2014). As Spurling and McMeekin (2014) suggest, this attention to recrafting practices is one of three possible strategies for creating sustainable mobility interventions, where the other two are substituting practices and changing how practices interlock. With the latter strategy, the focus lies more on the demand for mobility and reducing this in order to make the practice more sustainable. Bringing the negotiability of needs (Shove, 2003; Strengers, 2011) to the foreground, and the way mobility practices are related to interlocking practices, such as shopping and driving children to school, can be useful for creating sustainable mobility interventions (Spurling and McMeekin, 2014). For all three intervention strategies, it is relevant to understand the dynamics of practices. Social practices can for analytical purposes be deconstructed into several interconnected elements (Reckwitz, 2002). One helpful deconstruction tool is the three-element model, where practices are shaped by elements in the following categories: materials, competences and meanings (Shove et al., 2012). As Shove et al. (2012) explain, practice elements in all three categories must exist, as well as links between them, for people to be able to perform practices. Material elements are the technologies and tangible physical objects needed for the practice. Competences refer to the skills required for the performance of the practice and the embodied knowledge possessed by the carrier of the practice. Meanings comprise shared understandings of what is considered right and suitable by the many.

\section{Research setup}

In this section, we describe the CMaaS system that was implemented and the research methods used to study employees' experiences and understandings of it.

\subsection{The CMaaS system}

In this article we study the design, development and implementation of Corporate Mobility as a Service (CMaaS) at a large workplace outside Stockholm, Sweden. The corporate mobility service system (see Fig. 1) includes 40 e-bikes, three taxis, 14 shuttle buses and six commuter buses, the digital platform and the mobile application. The system is provided to 14,000 employees working in 70 buildings. The ebikes are provided at four stations. The shuttle buses cover an area that spans $5 \mathrm{~km}$ from north to south and $2 \mathrm{~km}$ from east to west, and is operated by three routes with 20 stops. The commuter bus has two stops in the city centre, $30 \mathrm{~km}$ from the industrial estate, and nine stops at the industrial estate. The company's industrial estate covers around four square kilometres, and facilities are also distributed over several sites in the town with distances between buildings up to $5 \mathrm{~km}$. For many years the company has provided internal taxi and shuttle bus services to assist employees in getting around the area for meetings. Since 2012, the company has also provided a commuter bus service to and from Stockholm city centre.

With the intention to explore sustainable urban transport solutions, a project was set up to improve the company's internal transport. With this development, the company switched from a combined mobility service to a CMaaS system. The main result of the project development was bundling the different internal transport modes into one service (see Fig. 1). The service is based on a digital platform with booking and real-time travel information and has a user-friendly mobile application, using real-time positioning of vehicles. The service was branded with a new name and a consistent graphic identity, and the three existing travel modes were expanded with forty shared electric bikes placed at four bike stations. The taxi service, and the timetables and routes of existing buses and shuttles, were left unchanged.

The new CMaaS system was launched in May 2018. The marketing campaign and resulting media coverage was more comprehensive than usual for this type of internal project. This proved to have a strong effect on the users' awareness of the new service, their expectations of it, and their understanding of the system.

\subsection{Research methods}

A service design approach was used to understand users' mobility practices, including travel needs and underlying meanings held around transport practices, such as company norms and users' beliefs. User perspectives were investigated in four iterative rounds of semi-structured interviews (see Table 1), with each round building on the findings of the last. All interviews were carried out individually with new participants in each round. For two of the rounds, interviews were booked with participants and trigger materials were used, while the other two rounds were carried out contextually, with random participants, during or in conjunction with travel. The first trigger material used was three different scenarios to discuss possible future development of the service system. The second trigger material used was a travel log book to capture experiences while using the internal transport service and also connecting external transport services. During the same time span, we kept up to date with the project development by taking part in several project group meetings, and after the launch, we also followed up on the company's usage statistics.

This article presents the results from the four rounds of interviews with a particular focus on the results from the fourth round, conducted one month after launch to explore users' experiences of the new CMaaS system and their understanding of the underlying concepts. Service design practice was used in the analysis, including visual methods, but was also supported by collecting and categorising answers using spreadsheets and data management software (NVivo). User experiences were the main focus of the analysis, using practices as a unit of analysis. The interview participants spanned from a few non-users and people

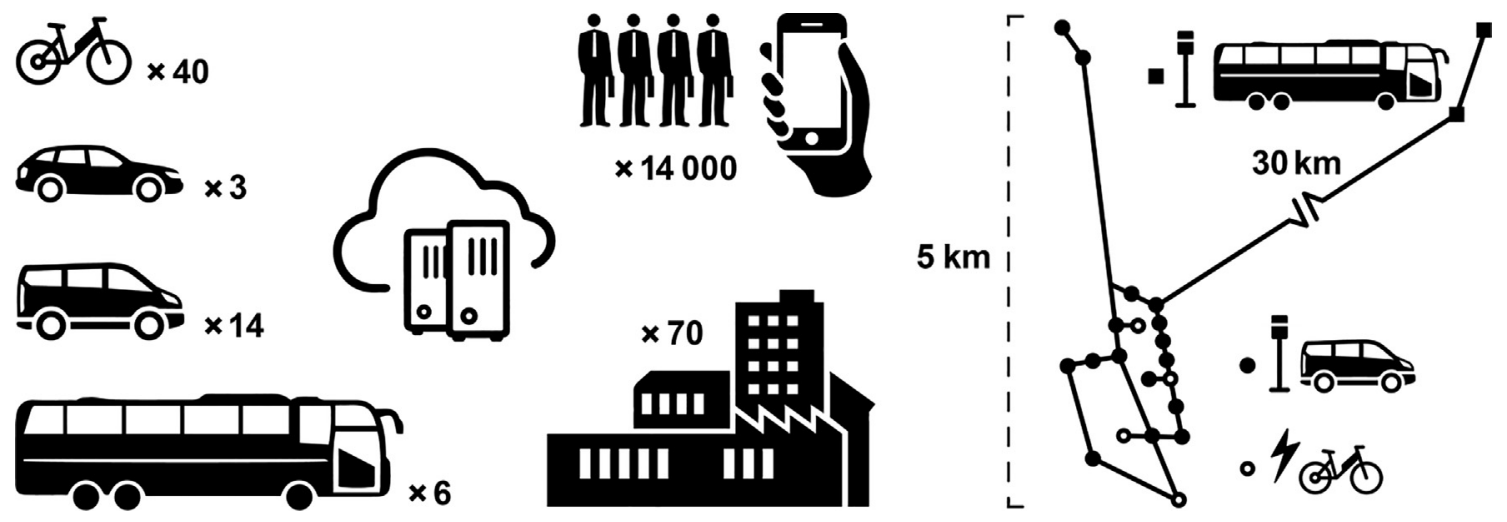

Fig. 1. The corporate mobility service system. 
who used the services very little, all the way to daily users (see interview details in Table 1).

\section{Results}

In this section, we present the outcomes from the implementation of CMaaS and the results from the interviews with users. First, we present some usage statistics from the first four months. This is followed by user insights from the interviews, concerning each of the system parts: service application, commuter bus service, shuttle bus service, taxi service and electric bike service.

\subsection{Usage statistics from the first four months}

The service application was downloaded by about 5000 employees in the first month, accounting for more than $30 \%$ of the employees. During the first four months it was used by on average 3300 unique devices per month, meaning that around $25 \%$ of the employees used the service application. On average, each user conducted around 9 sessions with the service application each month. Out of the 14,000 employees, 6000 were blue collar workers with mainly no transport needs during work hours but $20 \%$ of these indicated in a survey conducted by the company at the launch of the system a need to move once per day. Out of the remaining 8000 white collar workers, 55\% indicated that they travel around the area on a daily basis.

Usage of the commuter bus service, shuttle bus service and taxi service showed no significant changes in number of passengers over the four-month period after the launch. In total, the CMaaS system was used for around 25,000 passenger trips per month during the same period with an average of 11,100 passengers/month using the commuter bus, 10,800 passengers/month using the shuttle bus and 1480 passengers/month using the taxi service. The electric bike service was used by an average of 1620 passengers/month. On average, bike trips were about $2 \mathrm{~km}$ and lasted for about $19 \mathrm{~min}$ and on average 95 trips were conducted each day.

\subsection{Service application}

More than a third of employees downloaded the service application within the month after launch and about a quarter of them used it on a monthly basis. Employees appreciated the access to information regarding bus departures and timetables, for both the commuter and shuttle bus services. Timetables had been accessible previously, but the new features of real-time information and continuous updates about delays and changes were considered useful. During the first month, however, there were several problems with inaccurate tracking of vehicles, causing irritation among users. Also, as the service application was mainly built to support information on-the-go, some users expressed problems with planning their trips in advance (see first quote in Fig. 2). For example, if the service application presented three options for getting from point $\mathrm{A}$ to point $\mathrm{B}$ at a specific point in time, it was not possible for the user to see the whole timetable or to overview the complete route (see second quote in Fig. 2). The service application did include the possibility to view complete timetables, but for many this feature was hard to find. Hence, some users found that the application did not support decision making for planning their work days or future trips. These employees expressed a need for more or different information from the service application. Some employees indicated that they did not need the system at all - either they already knew the timetables or their specific bus departures by heart (see fourth quote in Fig. 2), or they found the frequency of buses sufficient to simply exit their building and wait for the next bus to arrive (see third quote in Fig. 2). These users saw no need to plan ahead. 


"In the SL-app [Stockholm public transport] you can select now or
tomorrow, or I want to be there at this or that time, but there is no such
function here, so I cannot plan anything in advance."
"I cannot get anything but here-and-now information."
"Since the shuttle buses are so frequent, I never need to, you know, I never
have to wait more than fifteen minutes anyway, so I haven't used the app."
"I know the company commuter bus timetable by heart."

Fig. 2. Quotes from interviews regarding the service application. Translated from Swedish to English by the authors.

\subsection{Corporate commuter bus service}

Employees who used the commuter bus service expressed appreciation of its convenience and comfort. For those who lived near to one of the two city pick-up points, or had easy access to public transport connecting to these locations, and who worked in buildings that were the first workplace stops, the commuter bus service was perceived as convenient. For these users, the service was almost door-to-door (see first quote in Fig. 3). For those who had to travel longer distances by public transport to get to the city stops, the service was considered less convenient. Also, those who worked in buildings located at the end of the commuter bus route regarded the service as less convenient as they felt they would have to spend half the commuting time travelling around the work site (see fourth quote in Fig. 3). Furthermore, some users felt that the somewhat limited number of departures was a restriction, or in some cases even a barrier to usage, for example when working late. Also, since the service had no booking facility, the limited seating capacity in some cases required users to queue ten minutes before departure time in order to secure a seat. This was especially the case for the morning departures at one of the city stops and when returning in the afternoons from one of the workplace stops. The users appreciated the comfort of the commuter bus, including spatial factors such as ergonomic seats, pleasant temperature (especially during cold winter months) and reduced noise levels, but also emotional factors like being able to spend commuting time with fellow colleagues and not with just any Stockholm commuter (who, according to respondents, could sometimes be noisy and disruptive). For some, especially those taller in height, there were complaints about restricted leg room and limited space for fold-up tables to be used for laptops in order to work on board. The possibility of working during the commuter bus trip was considered by many as a way of making commuting time useful, and some had even negotiated for this commuting time to be regarded as work hours with their managers. Others used the time on the bus for relaxation, to sleep or listen to music or audio books, in order to make commuting time valuable and less of a 'time thief' (see second quote in Fig. 3). When commuter bus users switched to commuting by car or public transport, the extra cost of the commuter bus service was often mentioned as not adding enough value (see third quote in Fig. 3). For these employees, the service's convenience and comfort did not provide enough value for money when considering the actual commuting time. Users who switched to commuting by car, a more expensive alternative, indicated flexibility as being more important. For those who switched to commuting by public transport, saving money or time was considered more important.

\subsection{Shuttle bus service}

Employees who had used the shuttle bus service regularly before the rebranded service was launched generally appreciated it more, both before and after the rebranding. With the redesigned vehicle exteriors and signage system for the bus stops, the various system parts became more visible and consistent, which was welcomed by employees (see first quote in Fig. 4). At the same time, some argued that the rebranding was only cosmetic and that no real improvements had been made. Some of the regular users of the shuttle bus had no choice but to use the internal transport system, as they used public transport for commuting to work. For car commuters, to use the shuttle bus service for internal transport instead of their private car meant that there was no need to find a parking space at their destination, e.g. the location of a meeting, or when returning to their workplace (see second quote in Fig. 4). This was considered an advantage as parking spaces were limited and finding them sometimes took a considerable amount of time. Those who did not use the service regularly often expressed doubts regarding its usefulness and thought it was inefficient. The service capacity in terms of routes and departure times was to some extent questioned and some felt that the limited capacity was a barrier to them using the service. The irregular departure times caused confusion and some referred to gaps in the timetables when the service was needed the most, such as in conjunction with the lunch hour when meetings were often booked to start straight after lunch. Furthermore, a couple of minutes' waiting time at a stop, to ensure synchronisation with the timetable, was considered annoying and inefficient. Many employees said that their workday was simply too packed with meetings and that time spent waiting for transport or standing idle at a bus stop was completely out of the question (see third quote in Fig. 4). The shuttle bus service not being connected, in either time or place, with public transport also

$$
\begin{aligned}
& \text { "It was really nice this last winter, to step inside the warm bus and get } \\
& \text { dropped off just outside the door." } \\
& \text { "I usually fall asleep, or, that is, you learn a trick or two over time." } \\
& \text { "It's half a monthly season ticket with SL [Stockholm public transport], } \\
& \text { so you should be able to demand more!" } \\
& \text { "You spend half the time sightseeing, going around the company area." }
\end{aligned}
$$

Fig. 3. Quotes from interviews regarding the commuter bus service. Translated from Swedish to English by the authors. 


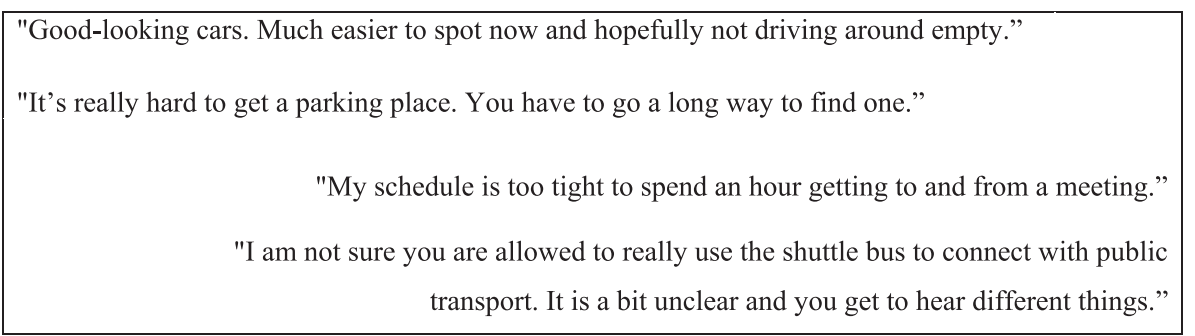

Fig. 4. Quotes from interviews regarding the shuttle bus service. Translated from Swedish to English by the authors.

caused frustration. Additionally, there were uncertainties about how to interpret the internal rules around the corporate mobility service system usage in conjunction with commuting trips (see fourth quote in Fig. 4).

\subsection{Taxi service}

The taxi service was considered helpful in terms of getting to locations not connected by the shuttle bus service. However, as the service was not bookable far in advance and to a set time but could only be used by phoning the switchboard to order a taxi immediately, it was felt to be unreliable (see fourth quote in Fig. 5). Some interviewees also reported that it took a long time to get to the switchboard to be able to book a taxi (see third quote in Fig. 5). Hence, the service was useful only when employees felt they had time to wait (see first quote in Fig. 5) or when they had no other travel options available (see second quote in Fig. 5). In these cases, for many, the alternative was to use their private car instead of the CMaaS system, or to share a ride with a colleague in their private car. Also, several employees expressed uncertainty about how to interpret the internal rules for when the taxi service should be used. Most felt it was clear that this service was intended only for meeting purposes, and that using it for private trips, for example to do physical exercise during lunch hour, would be illicit. However, if the physical exercise was carried out together with colleagues, for example playing floorball, and required transport between locations without access to the shuttle bus service, this was considered a grey zone in terms of what should be allowed for usage.

\subsection{Electric bike service}

The electric bike service was added as a new transport mode in the CMaaS system, and was highly appreciated by all employees interviewed (see first quote in Fig. 6). For employees based in one of the four buildings with bike stations, this service offered real advantages. Simple access to the bikes through the employee identification card allowed for flexibility. As the bikes could be used without pre-booking and at any time during work hours, there was no need to adjust one's planning to timetables (as required for the shuttle bus service) or waiting times (as needed for the taxi service). Instead, it was simply a matter of accessing a bike outside the office building. The service application made it possible to see how many bikes were currently accessible at each station and also how many parking spaces were available at the user's destination. Some employees reported that this increased flexibility enabled them to make full use of their time and they felt they were more time-

\footnotetext{
"When you have some slack in your schedule, this alternative is good."

"The internal taxi can be an alternative sometimes, like up to the Demo Centre."

"Sometimes it takes a lot of time, sometimes it's really quick. Sometimes it takes time just to get through to the switchboard."

"It would have been easier if I could just book it."
}

efficient when using the electric bike service than with other parts of the service system, or even compared to using their own cars (see second quote in Fig. 6). Additionally, there were employees who mainly appreciated that the electric bikes enabled them to get fresh air and relaxation time while moving between buildings. However, there were problems getting the system up and running, and during the first month there were several incidents with malfunctioning connectivity in the electric bike service system. Sometimes this prohibited employees from unlocking and using the bikes, causing frustration. Also, sometimes the electric bikes had been used so much during the day that they ran out of battery in the afternoon. When this occurred, even though there were bikes physically present, they were not available for usage. This caused irritation, and several employees questioned why the electric bikes could not be used as ordinary bikes in these cases. Also, the information provided in the service application regarding the number of bikes available was not always accurate, which caused uncertainty around the service. Furthermore, some said that limiting the electric bike usage to transport between the four designated buildings with parking stations prevented them from considering the bikes as a transport option. This was especially the case for those connecting with commuter trains or other public transport (see third quote in Fig. 6). However, the limited number of locations was also a contributing factor to not even consider using electric bikes when going to buildings without stations (see fourth quote in Fig. 6).

\section{Discussion}

In this section, we discuss the integration of the CMaaS system and the practices affected by redesigning the separate internal transport services and integrating them into one service. First, we discuss which mobility service practices emerged and what these consisted of. Second, we discuss how shared mobility practices were structured and how these particular practices were formed. Third, we discuss why sustainable mobility practices were obstructed and why these practices needed new and alternative linkages. In this section, we use social practice theories as lens to analyse the findings from the first four months' usage of the altered corporate mobility service system. This analytical lens consists of the three categories of elements - materials, competences and meanings - that are addressed in the following discussion, and we discuss how the practice perspective reveals integration needs for mobility service systems. 


"The electric bikes are great. Otherwise it's just the same as before."
"The simplicity and the freedom. Getting to meetings without even
having to think about timetables."
"It would be great if I could take an electric bike to the commuter train!'”,
"The simplest solution would be electric bike stations at each building."

Fig. 6. Quotes from interviews regarding the electric bike service. Translated from Swedish to English by the authors.

\subsection{Emerging mobility service practices}

During the four months after the launch of the redesigned system, employees used the pre-existing transport modes (commuter bus, shuttle bus and taxi) to more or less the same extent compared to the previous year. The new transport mode - the electric bikes - was used frequently and was highly appreciated by all interviewed employees. Thus, there were no significant changes in terms of usage of existing transport modes during the first four months, but there was increased usage of the system as a whole since one additional travel mode had been added. A dramatic increase in usage was not expected, as the redesigned system simply added a more consistent and attractive look and feel to the existing system components. However, the launch of the new service application with integrated and real-time information significantly affected employees' experiences and usage of information regarding the mobility system. It even caused new practices to emerge. Previously, information regarding the mobility system was accessed by employees through the corporate intranet service. This information contained timetables, including departure times and routes for the buses, but there were no frequent updates or disruption announcements available. The general experience of this information was that it was static and not always up-to-date or even relevant. Previously, information search was mainly conducted before the trips were carried out. Manual pre-trip planning was the only possible way to find information regarding the system. Hence, the prior system could not be considered a MaaS solution, as defined by Kamargianni et al. (2016) and others. With the new CMaaS system and the application made available for smartphones (both for iPhone and Android), the pre-trip planning could be complemented with wayside and on-board planning. Splitting the planning into these three stages can be useful when designing MaaS (Kamargianni et al., 2016), and to support information needs on-the-go was a central design principle in the development of the service application. However, experiences of the system were negatively affected by the fact that most employees were used to pre-trip planning, and the information in the application was not primarily structured for this. The employees had to re-learn how and when to access information which affected their mobility service practices.

The employees' transport practices for moving between locations at the work site primarily included transferring to different office buildings for meeting purposes, but also involved getting to lunch places and sports practice facilities within the work site during lunch hours. At the work site, the CMaaS system enabled these transport practices to be more sustainable than if employees were to use their own private fossilfuelled cars. However, the shuttle buses, taxis and electric bikes were still emitting more carbon dioxide than if employees had walked or cycled on regular bikes, which would be the most sustainable and healthiest mobility modes (Banister, 2008). However, these more sustainable options would require even more time for transport and therefore would be less time-efficient. The employees' internal transport practices (transport at the work site during, or in conjunction with, work hours) were to some extent structured by the corporate mobility service system. The employees who had not brought their own car to work and instead commuted by public transport were dependent on the CMaaS system for internal transport. Their only other options were to share rides with colleagues who had brought their cars, or to walk between locations. Those who had brought their cars were dependent on the CMaaS system to a lesser extent, but sometimes preferred using it anyway. Aside from dependency on the system, internal transport practices were also structured by the employees' lifestyle choices, where for example family and living situation highly influenced transport practices. As Spaargaren (2003) explains, social practices are constructed in the middle between lifestyles and systems of provision. Therefore, to understand how and why a practice develops, an understanding of both these sides is necessary. Systems of provision can enable people to live more sustainably, but they can also obstruct. In order to design such systems in a way that empowers more sustainable lifestyles to develop, attention must be paid to the dynamics of practices.

\subsection{Structuring shared mobility practices}

Understanding the dynamics of practices can be useful in order to promote less resource-demanding practices (Shove et al., 2007). As practices can be understood as structured by elements in the three categories of materials, competences and meanings (Shove et al., 2012), also the internal transport practices in this case can be analysed as formed by elements in these categories. In this section, we discuss how elements of the mobility service system within these three categories enabled the employees to structure their internal transport practices. In this discussion, the focus is on internal transport practices but as mentioned previously, these are also highly dependent on external transport practices, such as commuting practices.

\subsubsection{Material elements}

In the mobility service system, the material elements supporting internal transport practices were first of all the different vehicles, e.g. the commuter buses, shuttle buses, taxis and electric bikes. With the new and more visible graphic design, it was easier for employees to notice the vehicles and to be reminded of their existence. The material elements also included the service application with its interaction design aimed at facilitating information search on-the-go. The redesigned material elements emphasised and captured the company's vision to be a forerunner in the transformation towards sustainable transport. As such, the redesign was intentionally used as a strategic brand-building tool and this concretisation of the vision was highly appreciated by many employees. The added material elements, the service application and the electric bikes were crucial to the positive experiences of the system. The service application was frequently used by more than a quarter of the employees and it successfully supported wayside and onboard planning of trips, which previously had not been possible. The electric bikes were deeply appreciated as they provided flexibility and freedom that the previous system had lacked.

There were some negative comments regarding the comfort of the commuter bus vehicles, in terms of lack of space and noise levels, but there were also very positive comments regarding the same comfort issues, including a sense of exclusivity, as this part of the mobility system was only available to the employees. There were also issues with the system capacity, which was considered limited both in terms of frequency and routes. Optimising the system capacity, as a material 
element, is a research field on its own, and balancing frequency and routes with costs and risks is a delicate and strategic matter (AlonsoMora et al., 2017; Hyland and Mahmassani, 2018; Zhang et al., 2015).

When designing corporate mobility service systems, the material elements obviously need to be in place and they should be designed in line with user requirements. Without the material elements, such as the service application and the different transport modes, there will be no system, but introducing the material elements is not enough to form new mobility practices (Shove et al., 2012; Spurling and McMeekin, 2014; Urry, 2004). With mobility practices in focus, rather than the material system components, we also need to understand the required competences and shared meanings around the mobility practices.

\subsubsection{Competence elements}

The employees' internal transport practices also included elements that can be categorised as competences, such as the skills used to search for information and for travel planning. As mentioned earlier, the competences for pre-trip planning were already existing, whereas those for wayside and on-board planning had to be learned. This caused some problems, for example when employees tried to use the service application mainly for pre-trip planning. However, as employees discovered this alternative side of available information, there was also increasing appreciation. As this part of the practice was tried out, learned and mastered, it also became more valued.

In terms of the new material elements - the electric bikes - there were no issues with competence. Even though it turned out that some users had cycled without switching the electricity on, hence not attaining any extra help from the battery, users were still very happy with this mode of transport and thought it helped them to be more flexible than before. Some issues appeared regarding the booking of the taxi service: it was only available to book through the telephone switchboard, but some expected it to be bookable within the service application, and when this was not found possible, users perceived this part of the service inadequate. Also, the rules around use of the taxi service were not made explicit, and there were several interpretations of when and how to use it. Furthermore, the rules for how the shuttle buses could be used in connection to public transport were not clear. Some users understood that this connection was lacking, and not even allowed, because of tax reasons, whereas others had only heard rumours around why the shuttle buses were only available for transport between buildings for meeting purposes and not to access the train stations.

Competences to share resources at the workplace existed, where for example the use of department bicycles (that were not part of the mobility service system) was brought up in the interviews, as well as practices around sharing work spaces, meeting rooms and office facilities. Employees with access to department bikes described their practice of using them as a sharing practice. If they needed to park the bicycle for a longer period of time, for example during a long meeting in a different building, they did not feel that it was right to use it for that purpose. To make the most use out of the department bikes as a shared resource, these were only to be used for quick trips and errands, and not to be left idle at a location where nobody else could use them. This competence for sharing came out of a sense of contributing to resource efficiency, including physical resources, and being mindful of each other as colleagues, and sometimes also as friends.

\subsubsection{Meaning elements}

The meanings around the internal transport practices that supported use of the CMaaS system fell into three different categories: time efficiency, flexibility and convenience. First, employees appreciated that the system helped them to use their time efficiently. The system's purpose of facilitating and enabling quick and easy access to different locations for meetings around the work site was appreciated. Second, as employees with flexible work hours puzzled together their workdays, such as when and where to place meetings, where to start and finish work, and when to work from elsewhere, their life situations affected their travel practices. For them, flexibility also meant that they might need to pick up children from day care or carry out errands on the way home from work. Everyday life and work were described as intertwined, and employees found ways to use the system to support them in balancing their commitments. Third, the convenience of the system was to a large extent affected by its capacity and limitations. When the system's capacity was in line with employees' needs it supported usage and the development of the internal transport practice with the corporate mobility service system. The capacity was affected by frequency and routes for the bus services and by access points for the electric bike service.

There were also shared meanings amongst employees to the effect that the re-design and efforts invested in the CMaaS system were highly important as part of executing the company vision to strive towards more sustainable transport. Employees were proud of their employer leading the way, and appreciated the development and shared responsibilities. To share a vision and be part of its execution, even if only a small part of a big puzzle, was felt by many as satisfactory and important. Obviously, more contemporary systems have to be designed to support prevailing norms of time efficiency, flexibility and convenience (Shove et al., 2007), and also to fulfil the employees' expectations. But furthermore, when designing mobility service systems, it is important to enable shared meanings around its relevance and importance. To support sustainable mobility, policy regulations also need to include these visionary meanings, which could include more distant values and alternative benefits, in order to make them accepted (Banister, 2008).

\subsection{Obstructing sustainable mobility practices}

With the majority of the employees commuting by car, there is huge potential for change towards more sustainable mobility practices. However, the interviews revealed several obstructions to these possible sustainable developments. These barriers were found to be at three different integration levels: with external transport systems, corporate policy and norms, and societal regulations.

First, concerning external transport systems, the CMaaS system lacked integration such as combination tickets or including public transport in travel planning. Therefore, the internal transport was not fully integrated with other aspects of the users' commuting trips, and the system did not support the travel practice as a whole. A CMaaS needs to support both transport during work hours and commuting to and from work. A core function of Maas systems is the integration of different transport systems, supporting all types of travel and their connections.

Second, the corporate culture of how, when and where work is carried out significantly affects transport practices. With a strong meeting culture promoting face-to-face meetings and a leadership style of being physically present, many employees make frequent trips during the day. A more sustainable development could be to support more digital meetings and more flexible work situations (Spurling and McMeekin, 2014). These possibilities existed in some local teams, and some employees had negotiated special deals with their managers to work from home from time to time, or to count travel time as work hours, but these were not official corporate policies. Also, with high work pressure and stressful work situations followed less chance of shifting to more sustainable transport options. For more sustainable transport practices to emerge, and endure, at a workplace, these need to be supported by a CMaaS that provides for sustainable options. Furthermore, sustainable transport practices need to be supported by a corporate culture that promotes sustainable behaviour. Hence, corporate culture needs to be integrated with CMaaS and using the system needs to be part of the norm. Also, Maas systems need to be integrated with cultural and societal norms with their usage being perceived as the normal way of transport. For MaaS systems to become more sustainable, not only do they need to provide the more sustainable transport modes, such as bikes or electric vehicles, but they also need to support 
non-travel, for example working from home or at different locations through digital tools.

Third, many regulations regarding tax or the use of public spaces limit the potential of CMaaS to improve the overall travel situation for employees. Current Swedish tax regulations require employers to tax their employees if their commuting trips are subsidised. Therefore, the commuter bus service must be paid for by employees. At the same time, other regulations stipulate that car parking for employees should be free, if the municipality offers free parking in the same area. Also, the shuttle bus service could not fully connect with public transport, since company bus stops were not allowed to be placed next to train stations and public bus stops. Moreover, to clearly signal to Swedish tax authorities that there was no reason to tax subsidies for the employees, departure times were deliberately planned not to connect seamlessly. The same thinking was used when locating the electric bike pool stations which were located away from commuter train stations. Given current Swedish regulations, the CMaas could therefore not be integrated with public transport. Hence, regulations must be reformed to favour instead of discourage sustainable options. Integrating regulatory systems with CMaaS systems is not easy, as different stakeholders push in different directions. However, for Maas systems to succeed in developing sustainable mobility, this level of system integration is necessary.

As discussed, a practice perspective reveals integration needs for mobility service systems. However, in the study described in this article, the intention of the CMaaS system was to support internal transport. Therefore, the links between the internal and external transport are instead part of future developments and further analysis. Furthermore, analysis of sustainability aspects, such as possibly reduced levels of carbon dioxide emissions, are also part of future work. Possible system developments for further integration are necessary to reach the full potential of CMaas but are part of future work.

\section{Conclusions}

In this article, the concept of Corporate MaaS (CMaaS) has been introduced, and empirical results has been presented from studying CMaaS at a 14,000-employee workplace in Sweden. The CMaaS system was created by bundling three existing transport modes into one service with cohesive branding, a user-friendly interface, online booking and real-time travel information. In addition, a fourth transport mode, electric bikes, was added.

The research results show that the redesign of the separate transport services into one service was appreciated by employees, but also points to unfulfilled needs and also increased expectations from users. In the interviews, users requested better synchronisation between transport modes as well as integration with external systems, company policies and even corporate strategies. When designing MaaS systems, it is important to meet users' expectations, but also to understand the connected practices.

Changes in users' travel behaviours were (at the time of writing, after four months' operation) limited, and the only significant changes were due to the added electric bike mode. The two thirds of the employees that used to commute by car still took their car to work, since for most of them neither public transport nor the CMaaS system appeared to offer or support competitive alternatives for their commute. As a result, with the private car parked outside, many potential users never tried the CMaaS system, or only used it occasionally. This clearly indicates that inducing a switch to more sustainable transport modes requires more than adding a digital platform and a service application. There are opportunities for further research to gain a better understanding of the non-users, and how the use of both "carrots and sticks" could reduce car use or promote the use of more sustainable modes of transport.

In this article, social practices theories have been used as analytical lens to understand travel practices in the context of CMaaS. As CMaaS (and MaaS) are socio-technical systems, several perspectives must be integrated in the analysis. All system components, including materials (the user application, the transport modes, signage system), the competences (knowledge of how to use the materials), and the meanings (understandings of travel habits, lifestyle choices, and employer relations) must all be integrated for MaaS to support a reduction in the use of private cars.

In CMaaS, barriers related to MaaS implementation in terms of ticketing, pricing, and accessibility to the service are lowered. The learnings drawn from CMaaS about MaaS in general in this study are related to the practices of the users. Furthermore, through this lens of analysis, several opportunities and challenges for the adoption of CMaas and sustainable transport have been identified, all with relevance for MaaS in general. Three barriers to integration were identified at different levels: the need for integration with external transport systems; the need for integration with corporate policy, culture and norms that appear to be in conflict with using the services; and finally, society's laws and regulations that limit system development. To overcome these barriers, they must first be identified and included in the development work. This requires a better understanding of the users. However, this understanding must use an integrated perspective that includes people's work-life balance, of which their transport practices are only a part.

\section{Acknowledgements}

The project was partly funded by the Swedish research funding agency Vinnova within its Strategic Innovation Program Drive Sweden under grant number 2017-01976.

\section{References}

Alonso-Mora, J., Samaranayake, A., Wallar, E., Frazzoli, E., Rus, D., 2017. On-demand high-capacity ride-sharing via dynamic trip-vehicle assignment. Proc. Natl. Acad. Sci. 114 (3), 462-467.

Banister, D., 2008. The sustainable mobility paradigm. Transp. Policy 15 (2), 73-80. https://doi.org/10.1016/j.tranpol.2007.10.005.

Brendel, A. B., Mandrella, M., 2016. Information systems in the context of sustainable mobility services: a literature review and directions for future research. In: Twentysecond Americas Conference on Information Systems, San Diego, 2016 (pp. 1-10).

Cassell, C., Johnson, P., 2006. Action research: explaining the diversity. Human Relat. 59 (6), 783-814. https://doi.org/10.1177/0018726706067080.

Fishman, E., Washington, S., Haworth, N., 2014. Bike share's impact on car use: evidence from the United States, Great Britain, and Australia. Transport. Res. Part D 31, 13-20. https://doi.org/10.1016/j.trd.2014.05.013.

Giddens, A., 1991. Modernity and Self-identity: Self and Society in the Late Modern Age. Stanford University Press, Stanford, California.

Giesecke, R., Surakka, T., Hakonen, M., 2016. Conceptualising Mobility as a service. In Ecological Vehicles and Renewable Energies (EVER). In: 2016 Eleventh International Conference. IEEE, Monte Carlo, Monaco, pp. 1-11 https://doi.org/10.1109/ EVER.2016.7476443.

Ho, C.Q., Hensher, D.A., Mulley, C., Wong, Y.Z., 2018. Potential uptake and willingnessto-pay for Mobility as a Service (MaaS): a stated choice study. Transp. Res. A: Policy and Practice 117, 302-318. https://doi.org/10.1016/j.tra.2018.08.025.

Hyland, M., Mahmassani, H.S., 2018. Dynamic autonomous vehicle fleet operations: optimization-based strategies to assign AVs to immendiate travel demand requests. Transp. Res. Part C: Emerging Technologies 92, 278-297. https://doi.org/10.1016/j. trc.2018.05.003.

Jackson, T., 2005. Motivating sustainable consumption. Sustainable Devel. Res. Network $29,30$.

Jittrapirom, P., Caiati, V., Feneri, A.-M., Ebrahimigharehbaghi, S., González, M., Narayan, J., 2017. Mobility as a service: a critical review of definitions, assessments of schemes, and key challenges. Urban Plann. 2 (2), 13-25. https://doi.org/10.17645/ up.v2i2.931.

Kamargianni, M., Weibo, L., Matyas, M., Schäfer, A., 2016. A critical review of new mobility services for urban transport. Transp. Res. Procedia 14, 3294-3303. https:// doi.org/10.1016/j.trpro.2016.05.277.

Lu, M., Taiebat, M., Xu, M., Hsu, S.-C., 2018. Multiagent spatial simulation of autonomous taxis for urban commute: travel economics and environmental impacts. J. Urban Plann. Dev. 144 (4), 4018033. https://doi.org/10.1061/(ASCE)UP.1943-5444. 0000469.

Manzini, E., Vezzoli, C., 2003. A strategic design approach to develop sustainable product service systems: examples taken from the 'environmentally friendly innovation' Italian prize. J. Cleaner Prod. 11 (8), 851-857. https://doi.org/10.1016/S09596526(02)00153-1.

Martin, E., Shaheen, S.A., Lidicker, J., 2010. Impact of carsharing on household vehicle 
holdings: results from North American shared-use vehicle survey. Transp. Res. Record 2143 (1), 150-158. https://doi.org/10.3141/2143-19.

Matyas, M., Kamargianni, M., 2017. A stated preference experiments for mobility-as-aservice plans. In: 2017 5th IEEE International Conference on Models and Technologies for Intelligent Transportation Systems (MT-ITS). IEEE, Naples, Italy, pp. 738-743.

Matyas, M., Kamargianni, M., 2018. The potential of mobility as a service bundles as a mobility management tool. In: 97th Transportation Research Board Annual Meeting, 7th - 11th January 2018, Washington DC. https://doi.org/10.1007/s11116-0189913-4.

Pernestål Brenden, A., Hesselgren, M., Bauer, D., 2018. Possibilities in ride-sharing in work commuting - a case study in Sweden. In: Proceedings of 7th Transport Research Arena TRA 2018, April 16-19, 2018, Vienna, Austria.

Pernestål Brenden, A., Holmberg, P., 2017. Roadmap for action area combined mobility in Sweden Time perspective: 2017-2027, with focus on 2017-2020. Retrieved from https://kompis.me/wp-content/uploads/2018/01/roadmapp eng 10jan 2018-1.pdf.

Reckwitz, A., 2002. Toward a theory of social practices: a development in culturalist theorizing. Eur. J. Social Theory 5 (2), 243-263. https://doi.org/10.1177/ 13684310222225432

Sanders, L., 2008. An evolving map of design practice and design research. Interactions 15 (1), 13-17. https://doi.org/10.1145/1409040.1409043.

Sanders, L., Stappers, P.J., 2014. From designing to co-designing to collective dreaming: three slices in time. Interactions 21 (6), 24-33. https://doi.org/10.1145/2670616.

Shove, E., 2003. Comfort, Cleanliness and Convenience: The Social Organization of Normality. Berg, Oxford, UK.

Shove, E., Pantzar, M., Watson, M., 2012. The Dynamics of Social Practice: Everyday life and how it changes. SAGE Publications Ltd., London, UK https://doi.org/10.4135/ 9781446250655.

Shove, E., Watson, M., Ingram, J., 2007. Designing and Consuming: Objects, Practices and Processes.

Sochor, J., Karlsson, I.C.M., Strömberg, H., 2016. Trying out mobility as a service: experiences from a field trial and implications for understanding demand. Transp. Res. Rec. 2542 (2542), 57-64. https://doi.org/10.3141/2542-07.
Sopjani, L., Hesselgren, M., Ritzén, S., Janhager Stier, J., 2017. Co-creation with diverse actors for sustainability innovation. In: DS 87-8 Proceedings of the 21st International Conference on Engineering Design (ICED 17), vol 8: Human Behaviour in Design, Vancouver, Canada, pp. 459-468.

Spaargaren, G., 2003. Sustainable consumption : a theoretical and environmental policy perspective. Soc. Nat. Resour.: Int. J. 16, 687-701. https://doi.org/10.1080/ 08941920390217429.

Spurling, N., McMeekin, A., 2014. Interventions in practices: Sustainable mobility policie in England. In: Strengers, Y., Maller, C. (Eds.), Social Practices, Intervention and Sustainability: Beyond Behaviour Change. Routledge, pp. 78-94. https://doi.org/10. 4324/9781315816494.

Stickdorn, M., Schneider, J., 2014. This is Service Design Thinking: Basics - Tools - Cases. BIS Publisher, Amsterdam.

Strengers, Y., 2011. Negotiating everyday life: the role of energy and water consumption feedback. J. Consumer Culture 11 (3), 319-338. https://doi.org/10.1177/ 1469540511417994.

Strengers, Y., Maller, C., 2014. Social practices, intervention and sustainability: beyond behaviour change. Routledge.

UNEP, 2016. A Framework for Shaping Sustainable Lifestyles: Determinants and Strategies. DTI/1717/PA.

Urry, J., 2004. The 'system' of automobility. Theory, Cult. Soc. 21 (5), 25-39. https://doi. org/10.1177/0263276404046059.

Wilson, C., Pettifor, H., Cassar, E., Kerr, L., Wilson, M., 2018. The potential contribution of disruptive low-carbon innovations to $1.5{ }^{\circ} \mathrm{C}$ climate mitigation. Energy Effi. 1-18. https://doi.org/10.1007/s12053-018-9679-8.

Wong, Y.Z., Hensher, D.A., Mulley, C., 2017. Emerging transport technologies and the modal efficiency framework: a case for mobility as a service (MaaS). In: 15th International Conference on Competition and Ownership in Land Passenger Transport (Thredbo 15). Stockholm, Sweden.

Zhang, R., Spieser, K., Frazzoli, E., Pavone, M., 2015. Models, algorithms, and evaluation for autonomous mobility-on-demand systems. In: American Control Conference (ACC). IEEE, pp. 2573-2587. 